\title{
EU COST Action TD0801: Statistical Ghallenges On The 1000 Euro Genome Sequences In Plants
}

\author{
Marco C.A.M. Bink', Thomas Schiex ${ }^{2}$
}

'Biometris Wageningen UR, Wageningen, The Netherlands 'MIA - INRA Chemin de Borde Rouge, Castanet-Tolosan, France http://www.statseq.eu/

New DNA sequencing technologies either currently available or under development will eventually enable eukaryotic genomes to be sequenced for less than a thousand euros. This technologypush will have a major impact on plant genomics and biological research and lead to a dramatic expansion in both the availability of sequence data and the range of sequence based applications. New innovative techniques are required to unlock the information contained in the sequence data and to apply the acquired knowledge for plant science and crop improvement. The wide variety and often unique characteristics of plant genomes pose additional challenges and opportunities. The need for and the dissemination of efficient strategies for handling and analysing high throughput sequence data in plants requires cooperation at the international level to develop new approaches analytical tools and share best practice. This COST Action will establish a network of researchers that coordinate, focus and strengthen national and pan-European statistical genomics and bioinformatics. It will be built on close interactions with other disciplines such as genetics, genomics and breeding. The Working Groups will arrange workshops, Short Term Scientific Missions, a website and Wiki, training courses, and publications to disseminate aims and achievements.

\section{Relevant Web sites}

1. www.statseq.eu (COST Action TD0801)

2. https://colloque.inra.fr/statseg 2011/ ( $3^{\text {rd }}$ StatSeq workshop, Toulouse 2011)

3. www.bioinf.boku.ac.at/statseq (WG1 meeting on RNA seq, Vienna 2011) 